Review Article

\title{
Classifications for Proliferative Vitreoretinopathy (PVR): An Analysis of Their Use in Publications over the Last 15 Years
}

\author{
Salvatore Di Lauro, ${ }^{1,2}$ Mustafa R. Kadhim, ${ }^{3}$ David G. Charteris, ${ }^{3}$ and J. Carlos Pastor ${ }^{1,2}$ \\ ${ }^{1}$ IOBA (Eye Institute), University of Valladolid, 47011 Valladolid, Spain \\ ${ }^{2}$ Hospital Clínico Universitario de Valladolid, 47003 Valladolid, Spain \\ ${ }^{3}$ Moorfields Eye Hospital, London EC1V 2PD, UK \\ Correspondence should be addressed to Salvatore Di Lauro; sadilauro@live.it
}

Received 22 January 2016; Accepted 24 May 2016

Academic Editor: Stephen Charn Beng Teoh

Copyright (C) 2016 Salvatore Di Lauro et al. This is an open access article distributed under the Creative Commons Attribution License, which permits unrestricted use, distribution, and reproduction in any medium, provided the original work is properly cited.

Purpose. To evaluate the current and suitable use of current proliferative vitreoretinopathy (PVR) classifications in clinical publications related to treatment. Methods. A PubMed search was undertaken using the term "proliferative vitreoretinopathy therapy". Outcome parameters were the reported PVR classification and PVR grades. The way the classifications were used in comparison to the original description was analyzed. Classification errors were also included. It was also noted whether classifications were used for comparison before and after pharmacological or surgical treatment. Results. 138 papers were included. 35 of them (25.4\%) presented no classification reference or did not use any one. 103 publications (74.6\%) used a standardized classification. The updated Retina Society Classification, the first Retina Society Classification, and the Silicone Study Classification were cited in $56.3 \%, 33.9 \%$, and $3.8 \%$ papers, respectively. Furthermore, 3 authors (2.9\%) used modified-customized classifications and $4(3.8 \%)$ classification errors were identified. When the updated Retina Society Classification was used, only $10.4 \%$ of authors used a full C grade description. Finally, only 2 authors reported PVR grade before and after treatment. Conclusions. Our findings suggest that current classifications are of limited value in clinical practice due to the inconsistent and limited use and that it may be of benefit to produce a revised classification.

\section{Introduction}

Proliferative vitreoretinopathy (PVR) remains the major complication after retinal detachment surgery [1-3]. PVR was identified as an independent clinical entity in 1983 by the Retina Society Terminology Committee and a classification was created [4], based on the condition formerly named "massive vitreous traction" or "massive periretinal proliferation" [5-7]. This classification divided PVR into four stages, $A, B, C$, and $D$, apparently by increasing its severity, from minimal to massive PVR (Table 1). Nevertheless, this classification had numerous limitations. It did not consider the location of the vitreoretinal traction and the magnitude of the contraction. In addition, some of the stages provide a false idea of severity; for instance, D4 caused by a localized epiretinal membrane could be more easily treated by surgery than a Cl caused by intraretinal changes $[8,9]$. In 1989, the Silicone Study Group introduced a new classification [10] including some characteristics, such as the location, anterior or posterior, and the type of contraction (Table 2). Classification was then updated in 1991 [11] according to modifications proposed by the Silicone Study Group and also by other authors [12] (Table 3). This classification appears to be difficult to use in clinical practice and may not offer any special advantage for decision-making in relation to the treatment of the disease. Moreover, the classifications might have prevented advances in the understanding of the disease pathogenesis. For instance, because the Retina Society Committee defined PVR as a "proliferative disease," many treatments based on the inhibition of cell proliferation were developed for more than 20 years, none of which appears to have produced a significant clinical advance. Therefore, a review of both the classification and the pathogenesis of 
TABLE 1: Classification from the Retina Society Terminology Committee (1983) [4]. This classification subdivided PVR into four stages, A, B, $\mathrm{C}$, and D, apparently increasing in severity, from minimal to massive PVR. Modified from The Retina Society Terminology Committee, [4]

\begin{tabular}{ll}
\hline Grade & $\begin{array}{c}\text { Retina Society Terminology Committee (1983) } \\
\text { Clinical signs }\end{array}$ \\
\hline A (minimal) & Vitreous haze and pigment clumps \\
\hline B (moderate) & Surface retinal wrinkling, rolled edges of the retinal, retinal stiffness, and vessel tortuosity \\
\hline ( marked) & Full-thickness fixed retinal folds in \\
(i) C-1 & (i) one quadrant, \\
(ii) C-2 & (ii) two quadrants, \\
(iii) C-3 & (iii) three quadrants \\
\hline D (massive) & Fixed retinal folds in four quadrants that result in \\
(i) D-1 & (i) a wide funnel shape, \\
(ii) D-2 & (ii) a narrow funnel shape, \\
(iii) D-3 & (iii) closed funnel without view of the optic disc \\
\hline
\end{tabular}

TABLE 2: Silicone Study Classification (1989). This classification scheme included proliferative membranes in the preequatorial region and on the vitreous base (anterior PVR). Attention was paid to the quantitative assessment of PVR (number of clock hours of the retina involved by membranes). Minimal (grade A) and moderate (grade B) classifications remained unchanged, but grades C and D were replaced by grades $\mathrm{P}$ and A (posterior and anterior forms). Moreover, grades P and A were further defined by the presence of "types" of contraction. The extension of each grade was assessed by the number of clock hours of the involved retina. Modified from [10].

\begin{tabular}{|c|c|}
\hline \multicolumn{2}{|c|}{ Silicone Study Classification (1989) } \\
\hline Grade and type & Clinical signs \\
\hline A & Vitreous haze and pigment clumps \\
\hline B & $\begin{array}{l}\text { Surface retinal wrinkling, rolled edges of the retinal, retinal } \\
\text { stiffness, and vessel tortuosity }\end{array}$ \\
\hline $\begin{array}{l}\text { P } \\
\text { P1: } 1 \text { quadrant (1-3 clock hours) } \\
\text { P2: } 2 \text { quadrants ( } 4-6 \text { clock hours) } \\
\text { P3: } 3 \text { quadrants ( } 7-9 \text { clock hours) } \\
\text { P4: } 4 \text { quadrants (10-12 clock hours) } \\
\text { (i) Type } 1 \text { (focal) } \\
\text { (ii) Type } 2 \text { (diffuse) } \\
\text { (iii) Type } 3 \text { (subretinal) }\end{array}$ & $\begin{array}{l}\text { Starfolds and/or diffuse Contraction in posterior retina } \\
\text { and/or subretinal membrane in posterior retina } \\
\text { (i) Starfold } \\
\text { (ii) Confluent irregular retinal folds in posterior retina; } \\
\text { remainder of retina drawn posterior; optic disc that may not } \\
\text { be visible } \\
\text { (iii) "Napkin ring" around disc or "clothesline" elevation of } \\
\text { retina }\end{array}$ \\
\hline $\begin{array}{l}\text { A } \\
\text { A1: } 1 \text { quadrant (1-3 clock hours) } \\
\text { A2: } 2 \text { quadrants ( } 4-6 \text { clock hours) } \\
\text { A3: } 3 \text { quadrants ( } 7-9 \text { clock hours) } \\
\text { A4: } 4 \text { quadrants (10-12 clock hours) } \\
\text { (i) Type } 4 \text { (circumferential) } \\
\text { (ii) Type } 5 \text { (perpendicular) } \\
\text { (iii) Type } 6 \text { (anterior) }\end{array}$ & $\begin{array}{l}\text { Circumferential and/or perpendicular and/or anterior } \\
\text { traction in anterior retina } \\
\text { (i) Irregular retinal folds in the anterior retina; series of radial } \\
\text { folds more posteriorly; peripheral retina within vitreous base } \\
\text { stretched inward } \\
\text { (ii) Smooth circumferential fold of retina at insertion of } \\
\text { posterior hyaloids } \\
\text { (iii) Circumferential fold of retina at insertion of posterior } \\
\text { hyaloids pulled forward; trough of peripheral retina } \\
\text { anteriorly; ciliary processes stretched with possible hypotony; } \\
\text { iris retracted }\end{array}$ \\
\hline
\end{tabular}

Quadrants refer to the circumferential area of retina directly involved in contraction. One quadrant, 1-3 clock hours; two quadrants, 4-6 clock hours; three quadrants, 7-9 clock hours; and four quadrants, 10-12 clock hours. Clock hours need not necessarily be contiguous.

PVR appears to be appropriate to aid the development of new treatments [3].

There is a clinical impression that over the last 15 years clinicians have abandoned current PVR classifications. Thus, the purpose of this study has been to evaluate the current use of PVR classifications in papers dealing with clinical practice and therapy as an indirect measure of the degree of usefulness of existing classifications.

\section{Methods}

A search in PubMed for papers published between January 2000 and January 2014 was undertaken by two independent researchers. The basic term "proliferative vitreoretinopathy therapy" has been used. Inclusion criteria comprised human studies published in English, French, and Spanish on PVR or retinal detachment with specific mention to 
TABLE 3: The updated Retina Society Classification (1991). The revised classification incorporated changes proposed by the Silicone Study Group [10] and modifications proposed also by other authors [12]. Three grades of supposed increasing severity were described, emphasizing the posterior and anterior locations of proliferation. The new classification kept grades A and B, modified grade C, and eliminated grade D. According to the Silicone Study Classification, a more detailed description of grade C (posterior and anterior) PVR was made by adding the types of contraction, the extent of which was detailed by using clock hours instead of quadrants. Modified from [11].

\begin{tabular}{|c|c|}
\hline \multicolumn{2}{|c|}{ The updated Retina Society Classification [11] } \\
\hline Grade and type & Clinical signs \\
\hline A & $\begin{array}{l}\text { Vitreous haze, pigment clumps, and pigment clusters on inferior } \\
\text { retina }\end{array}$ \\
\hline B & $\begin{array}{l}\text { Wrinkling of inner retinal surface, retinal stiffness, vessel } \\
\text { tortuosity, rolled and irregular edge of retinal break, and decreased } \\
\text { mobility of vitreous }\end{array}$ \\
\hline $\begin{array}{l}\text { CP (posterior) } \\
\text { (i) Type: } \\
\text { (a) Focal } \\
\text { (b) Diffuse } \\
\text { (c) Subretinal }\end{array}$ & $\begin{array}{l}\text { Full-thickness retinal folds or subretinal strands posterior to } \\
\text { equator (1-12 clock hours involvement) } \\
\text { (i) Starfolds posterior to vitreous base } \\
\text { (ii) Confluent starfolds posterior to vitreous base; optic disc that } \\
\text { may not be visible } \\
\text { (iii) Proliferation under the retina; annular strand near disc; linear } \\
\text { strands; moth-eaten-appearing sheets }\end{array}$ \\
\hline
\end{tabular}

Full-thickness retinal folds or subretinal strands anterior to equator (1-12 clock hours involvement), anterior displacement, and

CA (anterior)

(i) Type:

(a) Circumferential

(b) Anterior condensed vitreous strands

(i) Retina contraction inwards at the posterior edge of the vitreous base; with central displacement of the retina; peripheral retina stretched; posterior retina in radial folds

(ii) Snterior contraction on the retina at the vitreous base; ciliary body detachment and epiciliary membrane; iris retraction
PVR. Prospective randomized and nonrandomized clinical studies, retrospective clinical studies, short series, and pilot studies were included. A manual search of related articles was also performed through references reported in each article. Outcome parameters were reported PVR classification, if any, and reported PVR grades. We also analyzed how the different authors interpreted the classifications, comparing the results they provided with the original description. Therefore, we investigated if they used all the characteristics (grade [A, B, C], PVR localization, extension in hours, and type) of the updated Retina Society Classification or if they were used only partially. Classification errors were also included. Furthermore, it was also noted whether classifications were used both before and after an eventual PVR pharmacological or surgical treatment. Data were extracted using a custommade data sheet performed with agreement between authors.

\section{Results}

Preliminary search identified a total of 219 publications, 81 of which were eliminated as they did not fulfill the inclusion criteria. Thus, 138 publications were finally included and analyzed. 35 articles $(25,4 \%)$ did not undergo detailed analysis because there was no reference to the type of PVR classification used by the authors or because no classification was used (generic terms such as initial or severe PVR or simply PVR were adopted, without using any grading system). 103 publications $(74,6 \%)$ used standardized PVR classifications and they were analyzed in detail. The most used classification was the updated Retina Society Classification [11] (58 cites;
$56,3 \%)$, followed by the first Retina Society Classification [4] (35 cites; 33,9\%). 4 publications $(3,8 \%)$ used the Silicone Study Classification (Table 4) [10]. In addition, 3 authors $(2,9 \%)$ used modified or customized classifications [13-15]. In 4 publications $(3,8 \%)$, errors in the stated classification were identified $[13,16-18]$ : in two papers $[16,18]$, the authors cite the updated Retina Society ('91) but in fact they used the first Retina Society Classification ('83), Koerner et al., after citing the updated Retina Society Classification used a customized one [13], and Roldán-Pallarés et al. cite the two Retina Society Classifications at the same time [17].

Among the papers using the updated Retina Society Classification, the most documented was grade C PVR (48 articles, 83\%), while grades B and A were less frequently documented (15 (26\%) and 7 (12\%) articles, resp.). Of the publications documenting only $\mathrm{C}$ grade, the classification of subtypes was infrequently used. In 5 of 48 publications $(10,4 \%)$, there was a full $\mathrm{C}$ grade description in terms of localization (anterior or posterior), extension (in clock hours), and type (focal, diffuse, subretinal, circumferential, or anterior displacement). A similar finding was observed when authors used the first Retina Society Classification (35 papers): only $\mathrm{C}$ and $\mathrm{D}$ grades were frequently reported (27 and 10 papers, resp.), while grades $A$ and $B$ were infrequently used (4 and 7 articles, resp.). When the Silicone Study Classification was used, only grade $\mathrm{C}$ was mentioned (in 2 out of 4 articles). Finally, there were 2 publications $(1,9 \%)$ in which the standard classifications were used to assess changes in PVR status after any treatment, reporting PVR grade before and after it $[19,20]$. The remaining authors used the classifications only 
TABLE 4: Distribution of the used classifications for each year in papers published between January 2000 and January 2014. No data are shown about 2014 because there were no papers published at this time.

\begin{tabular}{lccc}
\hline Year of publication & $\begin{array}{c}\text { Retina Society } \\
\text { Classification } \\
(1983)\end{array}$ & $\begin{array}{c}\text { Silicone Study } \\
\text { Classification } \\
(1989)\end{array}$ & $\begin{array}{c}\text { Updated Retina } \\
\text { Society } \\
\text { Classification } \\
(1991)\end{array}$ \\
\hline 2000 & 4 & 1 & 0 \\
2001 & 1 & 0 & 3 \\
2002 & 6 & 0 & 2 \\
2003 & 2 & 0 & 6 \\
2004 & 1 & 0 & 4 \\
2005 & 5 & 0 & 1 \\
2006 & 4 & 0 & 7 \\
2007 & 1 & 1 & 1 \\
2008 & 3 & 0 & 6 \\
2009 & 0 & 0 & 10 \\
2010 & 3 & 0 & 2 \\
2011 & 2 & 0 & 0 \\
2012 & 1 & 1 & 6 \\
\hline
\end{tabular}

to describe PVR status, rather than using them to analyze the improvement or worsening of the previous stage after the applied treatment.

\section{Discussion}

Classifications of PVR were developed to provide clinicians with a useful tool to compare results of treatments. They are, to date, purely descriptive and do not reflect the pathobiology of this complex vitreoretinal disease. The original classification of 1983 [4], which was relatively simple to use, induces errors in the estimating severity in some cases. As mentioned, D grades were based on the ophthalmoscopic appearance of the detached retina, and experience demonstrated that some of these cases could be relatively easily solved by peeling localized epiretinal membranes although, in some cases, classified as $\mathrm{C}$, intraretinal severe changes prevent reattachment, unless complicated surgical techniques were used. Subsequent classifications $[10,11]$ were more detailed and therefore more complicated to use on a routine basis. They incorporated the anterior PVR forms, which add severity to the case, but until now no one has incorporated the intraretinal changes, observed in many surgical retinal samples $[8,9]$, which may prevent the anatomical reattachment of the retina and require retinectomy. Another important limitation is that they do not provide information on the activity of the process, although it has been observed that PVR progresses through several stages [1-3]. This may be crucial in estimating the risk of reproliferation after surgery or when surgeons schedule removing silicone oil, and no data on the chronology of events is included in any classification. Furthermore, current classifications are not prognostic and do not correlate with visual prognosis or anatomical success after surgical treatment.
Moreover, some of these classifications can be difficult to use in clinical practice, due to their complexity (grade, localization, extension, type, etc.) and because they may not provide useful information they have been largely abandoned by clinicians. Our findings in this study demonstrate that, in clinical research, investigators are using them inconsistently and reporting limited observations of stages (mostly grade $\mathrm{C}$ ).

The results of this study suggest that the current classifications are of limited value. Some authors simply do not use any, preferring generic terms, such as minimal, moderate, and severe PVR [21, 22]. Furthermore, early stages of PVR (named as grades $\mathrm{A}$ and $\mathrm{B}$ ), which are common to all classifications, are not used, and most authors refer only to most advanced stages, basically grade C $[20,23]$. Moreover, the presence of classification errors could indicate that there is confusion concerning their use $[13,16-18]$. An important relevant finding is that only a limited number of clinicians appropriately and fully use the current updated classification of 1991 [2427], while the majority of authors avoid using the added characteristics of localization, extension, and type, limiting the description to the grade. In addition, many colleagues still use the first reported classification [4], probably because although it presents many limitations, it is easier to use compared to the last version [23, 28]. Additionally, some authors decided to use alternative classification, probably to avoid problems evaluating PVR stages [14].

The use of multiple classifications makes the efficient communication between clinicians and the comparison of different studies very problematic. As mentioned, one of the aims of this study was to evaluate the usefulness of these classifications for PVR management with surgery and other adjunctive treatments. Unfortunately, many ophthalmologists did not use the normalized classifications for comparing 
the outcomes using non-clearly defined terms such as PVR recurrence $[29,30]$.

\section{Conclusions}

Our findings showed the inconsistent and limited use of the current PVR classifications suggesting that it may be of benefit to produce a revised classification incorporating, if possible, the new knowledge on PVR which has been published since 1991, pointing out new potential targets for therapeutic agents distinct from those, mainly proliferative agents, targeted by the original description of this disease. Thus, it is possible that we could reduce its prevalence after retinal detachment surgery and to improve the anatomical and functional results of this disease which resists the attempts of both basic researchers and clinicians for more than 30 years.

\section{Competing Interests}

The authors declare that they have no competing interests.

\section{References}

[1] J. C. Pastor, E. R. de la Rúa, and F. Martín, "Proliferative vitreoretinopathy: risk factors and pathobiology," Progress in Retinal and Eye Research, vol. 21, no. 1, pp. 127-144, 2002.

[2] J. C. Pastor, "Proliferative vitreoretinopathy: an overview," Survey of Ophthalmology, vol. 43, no. 1, pp. 3-18, 1998.

[3] J. C. Pastor, J. Rojas, S. Pastor-Idoate, S. Di Lauro, L. GonzalezBuendia, and S. Delgado-Tirado, "Proliferative vitreoretinopathy: a new concept of disease pathogenesis and practical consequences," Progress in Retinal and Eye Research, vol. 51, pp. 125-155, 2016.

[4] G. Hilton, R. Machemer, R. Michels, E. Okun, C. Schepens, and A. Schwartz, "The classification of retinal detachment with proliferative vitreoretinopathy," Ophthalmology, vol. 90, no. 2, pp. 121-125, 1983.

[5] W. H. Havener, "Massive vitreous retraction," International Ophthalmology Clinics, vol. 16, no. 1, pp. 135-155, 1976.

[6] R. Machemer, "Pathogenesis and classification of massive periretinal proliferation," British Journal of Ophthalmology, vol. 62, no. 11, pp. 737-747, 1978.

[7] J. D. Scott, "The treatment of massive vitreous retraction by the separation of pre-retinal membranes using liquid silicone," Modern Problems in Ophthalmology, vol. 15, pp. 185-190, 1975.

[8] J. C. Pastor, M. C. Méndez, M. A. de la Fuente et al., "Intraretinal immunohistochemistry findings in proliferative vitreoretinopathy with retinal shortening," Ophthalmic Research, vol. 38, no. 4, pp. 193-200, 2006.

[9] D. G. Charteris, J. Downie, G. W. Aylward, C. Sethi, and P. Luthert, "Intraretinal and periretinal pathology in anterior proliferative vitreoretinopathy," Graefe's Archive for Clinical and Experimental Ophthalmology, vol. 245, no. 1, pp. 93-100, 2007.

[10] J. S. Lean, W. H. Stern, A. R. Irvine et al., "Classification of proliferative vitrereotinopathy used in the silicone study," Ophthalmology, vol. 96, no. 6, pp. 765-771, 1989.
[11] R. Machemer, T. M. Aaberg, H. M. Freeman, A. R. Irvine, J. S. Lean, and R. M. Michels, "An updated classification of retinal detachment with proliferative vitreoretinopathy," American Journal of Ophthalmology, vol. 112, no. 2, pp. 159-165, 1991.

[12] K. Heimann and P. Wiedemann, "Cologne classification of proliferative vitreoretinopathy," in Proliferative Vitreoretinopathy, K. Heimann and P. Wiedemann, Eds., pp. 148-149, Kaden, Heidelberg, Germany, 1989.

[13] F. Koerner, U. Koerner-Stiefbold, and J. G. Garweg, "Systemic corticosteroids reduce the risk of cellophane membranes after retinal detachment surgery: a prospective randomized placebocontrolled double-blind clinical trial," Graefe's Archive for Clinical and Experimental Ophthalmology, vol. 250, no. 7, pp. 981-987, 2012.

[14] S. Schröder, P. S. Muether, A. Caramoy et al., "Anterior chamber aqueous flare is a strong predictor for proliferative vitreoretinopathy in patients with rhegmatogenous retinal detachment," Retina, vol. 32, no. 1, pp. 38-42, 2012.

[15] M. A. Acar, N. Ünlü, D. Hazirolan, M. Necati Demir, G. O. Üney, and F. Örnek, "Conventional surgery for complicated retinal detachment in silicone oil-filled eyes," European Journal of Ophthalmology, vol. 21, no. 3, pp. 290-295, 2011.

[16] S. Zenoni, N. Comi, P. Fontana, and M. R. Romano, “The combined use of heavy and light silicone oil in the treatment of complicated retinal detachment with $360^{\circ}$ retinal breaks: tamponade effect or filling effect?" Annals of the Academy of Medicine Singapore, vol. 41, no. 10, pp. 440-443, 2012.

[17] M. Roldán-Pallarés, C. Bravo-Llatas, A.-S. Musa, J. HernandezMontero, and R. Fernández-Durango, "Modelling postoperative visual acuity with and without proliferative vitreoretinopathy associated with primary rhegmatogenous retinal detachment," Eye, vol. 26, no. 10, pp. 1329-1336, 2012.

[18] T. Oyagi and K. Emi, "Vitrectomy without scleral buckling for proliferative vitreoretinopathy," Retina, vol. 24 , no. 2, pp. $215-$ 218, 2004.

[19] Z. Kapran, N. Acar, T. Altan, Y. B. Unver, and S. Yurttaser, "25gauge sutureless vitrectomy with oblique sclerotomies for the management of retinal detachment in pseudophakic and phakic eyes," European Journal of Ophthalmology, vol. 19, no. 5, pp. 853860, 2009.

[20] S. Auriol, V. Pagot-Mathis, L. Mahieu, C. Lemoine, and A. Mathis, "Efficacy and safety of heavy silicone oil densiron $68^{\circledR}$ in the treatment of complicated retinal detachment with large inferior retinectomy," Graefe's Archive for Clinical and Experimental Ophthalmology, vol. 246, no. 10, pp. 1383-1389, 2008.

[21] M. M. Lai, A. J. Ruby, R. Sarrafizadeh et al., "Repair of primary rhegmatogenous retinal detachment using 25-gauge transconjunctival sutureless vitrectomy," Retina, vol. 28 , no. 5, pp. 729-734, 2008.

[22] L. B. Oliveira and P. A. C. Reis, "Silicone oil tamponade in 23gauge transconjunctival sutureless vitrectomy," Retina, vol. 27, no. 8, pp. 1054-1058, 2007.

[23] M. T. Kralinger, U. Stolba, M. Velikay et al., "Safety and feasibility of a novel intravitreal tamponade using a silicone oil/acetylsalicylic acid suspension for proliferative vitreoretinopathy: first results of the Austrian Clinical Multicenter Study," Graefe's Archive for Clinical and Experimental Ophthalmology, vol. 248, no. 8, pp. 1193-1198, 2010.

[24] K. A. G. Shalaby, "Relaxing retinotomies and retinectomies in the management of retinal detachment with severe proliferative 
vitreoretinopathy (PVR)," Clinical Ophthalmology, vol. 4, no. 1, pp. 1107-1114, 2010.

[25] M. Mura, S. H. Tan, and M. D. De Smet, "Use of 25-gauge vitrectomy in the management of primary rhegmatogenous retinal detachment," Retina, vol. 29, no. 9, pp. 1299-1304, 2009.

[26] J. R. Minarcik and M. A. von Fricken, "Virtual retinectomy: indocyanine green-assisted internal limiting membrane peeling as a surgical adjunct in repair of recurrent rhegmatogenous retinal detachment due to PVR," Clinical Ophthalmology, vol. 6, no. 1, pp. 631-636, 2012.

[27] D. G. Charteris, G. W. Aylward, D. Wong, C. Groenewald, R. H. Y. Asaria, and C. Bunce, "A randomized controlled trial of combined 5-fluorouracil and low-molecular-weight heparin in management of established proliferative vitreoretinopathy," Ophthalmology, vol. 111, no. 12, pp. 2240-2245, 2004.

[28] W. Chen, H. Chen, P. Hou, A. Fok, Y. Hu, and D. S. C. Lam, "Midterm results of low-dose intravitreal triamcinolone as adjunctive treatment for proliferative vitreoretinopathy," Retina, vol. 31, no. 6, pp. 1137-1142, 2011.

[29] B. L. Lim and B. Vote, "Densiron intraocular tamponade: a case series," Clinical and Experimental Ophthalmology, vol. 36, no. 3, pp. 261-264, 2008.

[30] F. Boscia, C. Furino, N. Recchimurzo, G. Besozzi, G. Sborgia, and C. Sborgia, "Oxane HD vs silicone oil and scleral buckle in retinal detachment with proliferative vitreoretinopathy and inferior retinal breaks," Graefe's Archive for Clinical and Experimental Ophthalmology, vol. 246, no. 7, pp. 943-948, 2008. 


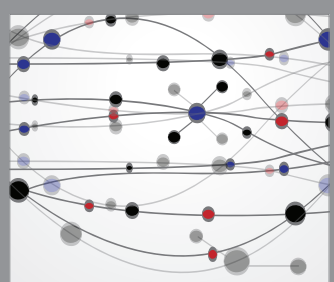

The Scientific World Journal
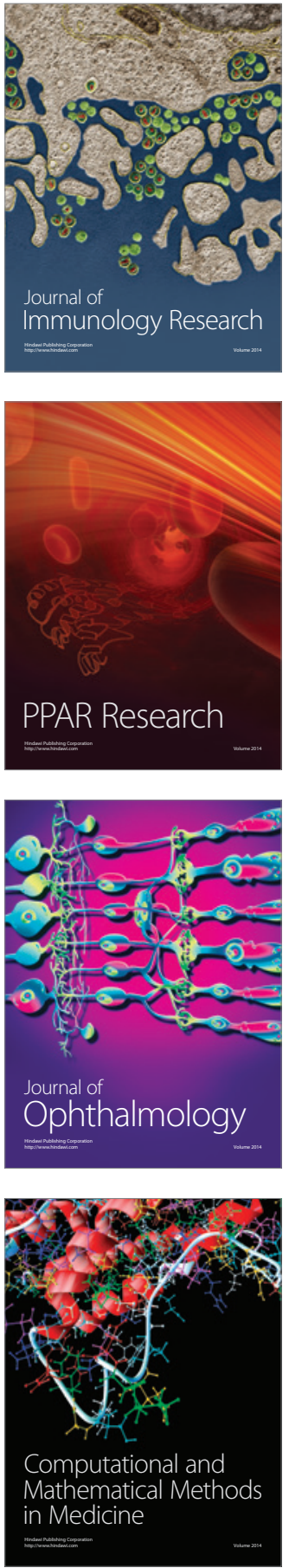

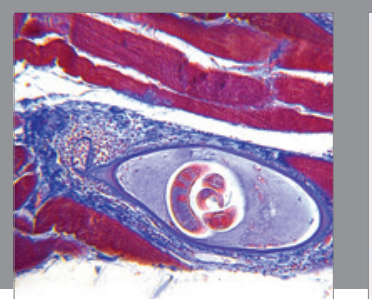

Gastroenterology Research and Practice

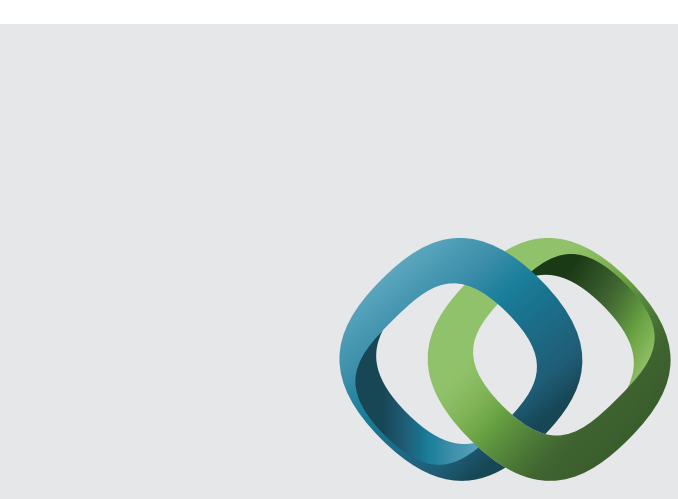

\section{Hindawi}

Submit your manuscripts at

http://www.hindawi.com
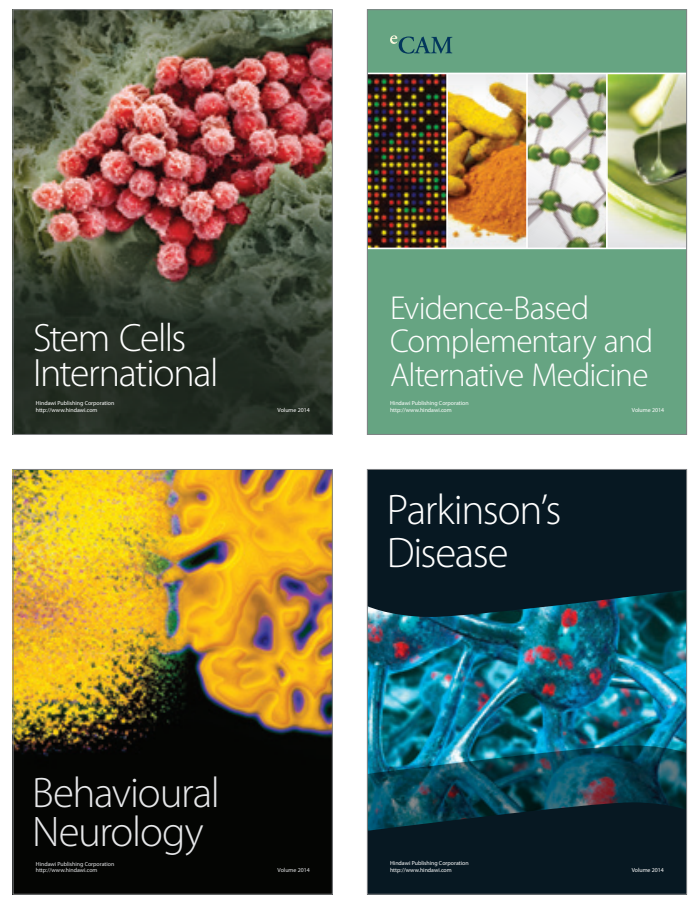
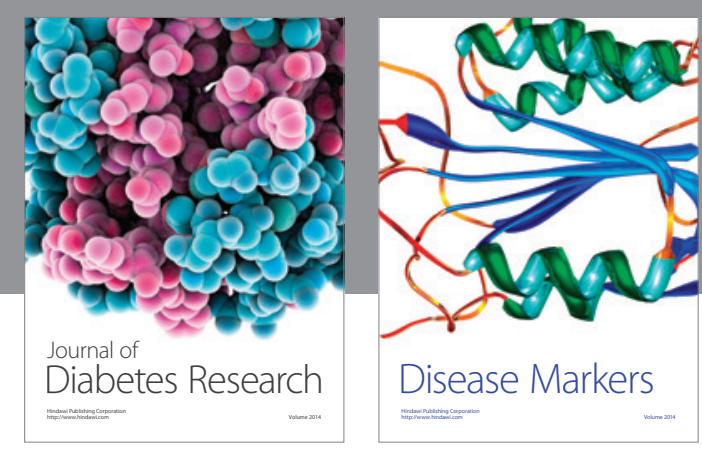

Disease Markers
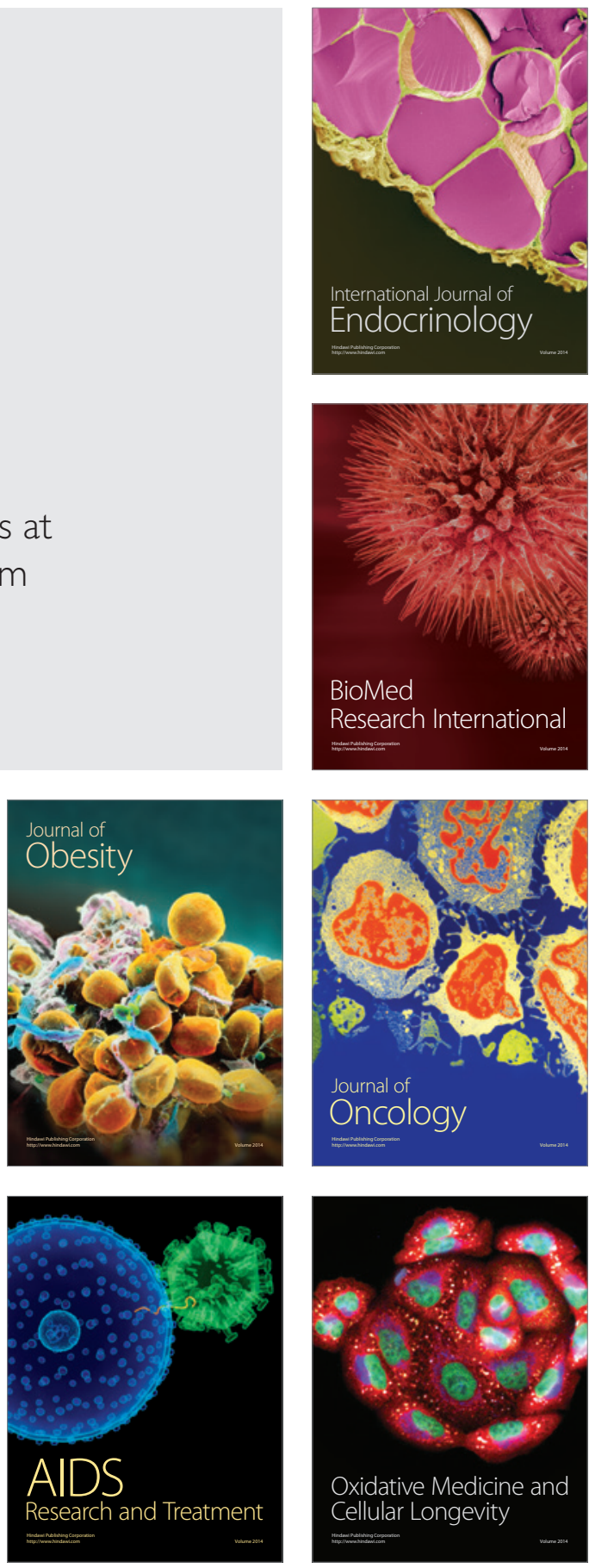